\title{
EVALUACIÓN DE LA COMPOSICIÓN FISICO-QUIMICA Y ETIQUETADO NUTRMENTAL DE LECHE ENTERA PASTEURIZADA COMERCIAL Y SU CUMPLIMIENTO CON LA NORMATIVIDAD OFICIAL MEXICANA
}

\author{
Evaluation of physicochemical composition and labeling information of commercial \\ pasteurized milk and their Mexican official regulatory compliance
}

Joshua Ángel Rizo Plascencia
Departamento de Ciencias Pecuarias y Agrícola. Centro Universitario de los Altos, Universidad de
Guadalajara. Av. Rafael Casillas Aceves 1200, 47600 Tepatitlán de Morelos, Jalisco, México.
Zuamí Villagrán de la Mora
Repartamento de Ciencias de la Salud. Centro Universitario de los Altos, Universidad de Guadalajara . Av.
Rafael Casillas Aceves 1200, 47600 Tepatitlán de Morelos, Jalisco, México.
Javier Eugenio García de Alba Verduzco
Departamento de Ciencias Pecuarias y Agrícola. Centro Universitario de los Altos, Universidad de
Guadalajara. Av. Rafael Casillas Aceves 1200, 47600 Tepatitlán de Morelos, Jalisco, México.
Blanca Catalina Ramírez Hernández
Departamento de Ciencias Pecuarias y Agrícola. Centro Universitario de los Altos, Universidad de
Guadalajara. Av. Rafael Casillas Aceves 1200, 47600 Tepatitlán de Morelos, Jalisco, México.
Erika Alvarado Loza
Departamento de Ciencias Pecuarias y Agrícola. Centro Universitario de los Altos, Universidad de
Guadalajara. Av. Rafael Casillas Aceves 1200, 47600 Tepatitlán de Morelos, Jalisco, México.
María Dolores Méndez Robles
Departamento de Ciencias Pecuarias y Agrícola. Centro Universitario de los Altos, Universidad de
Guadalajara. Av. Rafael Casillas Aceves 1200, 47600 Tepatitlán de Morelos, Jalisco, México
Laura Elena Iñiguez Muñoz
*ivisión de Ciencias Exactas Naturales y Tecnológicas. Centro Universitario del Sur, Universidad de
Guadalajara. Av. Enrique Arreola Silva 883, 49000, Ciudad Guzmán, Jalisco.
Luis Miguel Anaya Esparza

\section{Resumen}

En la actualidad, existe gran variedad de marcas de leche entera pasteurizada que se expenden en supermercados o tiendas de abarrotes. El objetivo de este trabajo fue evaluar la composición físico-química y el etiquetado nutrimental de leche de leche entera pasteurizada (48 muestras) comercial y su cumplimiento con la normatividad oficial mexicana. En general, todas las marcas cumplen con la información comercial requerida en la normatividad. Asimismo, las diferentes marcas de leche presentaron diferencias $(p<0.05)$ entre lotes de producción (excepto la marca $\mathrm{E}, p>0.05)$ y entre marcas $(p<0.05)$ con respecto a composición fisicoquímica. Por otro lado, ninguna marca presentó el contenido de proteína, grasa y lactosa reportado en su etiqueta $(p<0.05)$ ni con lo estipulado en la NOM-151-SCFI-2012. No obstante, de los parámetros de calidad que considera la normatividad, los porcentajes de cumplimiento de las marcas de leche evaluadas fueron $<40 \%$. Adicionalmente, el análisis clúster permitió agrupar la calidad físico-química de las marcas de leche en 4 grupos, el grupo 1 conformado por la marca A, el grupo 2 por la marca D, mientras que, en el grupo 3 se encontraron las marcas B y C y en el grupo 4 las marcas E, F, G y H. En este contexto, se requiere mayor vigilancia por parte de las autoridades correspondientes hacía las empresas del ramo, para asegurar el cumplimiento de los parámetros establecidos por la normatividad oficial vigente y permitan al consumidor obtener productos lácteos de buena calidad.

Palabras clave: Calidad, contenido nutrimental, etiquetado, cumplimiento normativo. 


\begin{abstract}
Currently, there is a wide variety of pasteurized milk brands sold in supermarkets and grocery stores. The objective of this study was to evaluate the physicochemical composition and nutritional information of commercial pasteurized milk commercial and their compliance with the official Mexican standards. In general, all milk brands comply with the commercial information required by the regulations. Likewise, the different pasteurized milk brands showed statistical differences between production batches of the same brand $(p<0.05)$ and milk brands $(p<0.05)$ in terms of physicochemical composition. On the other hand, any milk brands did not comply with the protein, fat, and lactose content reported in its labeling $(p<0.05)$ and the NOM-151SCFI-2012 stipulations. Additionally, the cluster analysis permits the aggrupation of milk brand quality in four groups. The first group is formed by the A sample, group 2 by the B sample, while, in the third group, the B and C samples, and the fourth group is characterized by the E, F, G, and $\mathrm{H}$ samples. In this context, greater vigilance by the corresponding authorities is required to ensure compliance with the parameters established by the official regulations and allow consumers to obtain good quality dairy products.
\end{abstract}

Keywords: Quality, labeling, nutritional content, regulatory compliance.

\section{Introducción}

Debido a su alto valor biológico, la leche y los subproductos lácteos son ampliamente consumidos por grandes segmentos de la población durante todas las etapas de la vida (Nicolau et al., 2011). La venta de productos lácteos en México representa uno de los sectores de mayor importancia dentro de la industria alimentaria. De acuerdo con la Dirección de Investigación y Evaluación Económica y Sectorial (DIEES, 2019), el consumo nacional de leche fluida en el año 2018 fue de 122 litros por persona, lo que equivale a $350 \mathrm{~mL}$ por día.

De acuerdo con la Cámara Nacional de la Industria de la Leche (2019) existen 20 empresas formales registradas, donde se elaboran leches pasteurizadas y ultra pasteurizadas, leches industrializadas, fórmulas infantiles, quesos, yogurt, cremas, mantequillas, helados y postres. Por su parte, el $30 \%$ de la leche fluida que se produce en México se destina a la manufactura de leche pasteurizada, homogenizada y ultra pasteurizada (CANILEC, 2021). La NOM-155-SCFI-2012 menciona que la leche pasteurizada es aquella sometida a un tratamiento térmico $\left(72^{\circ} \mathrm{C} / 15 \mathrm{~s}\right)$ que garantice la inocuidad del producto mediante la inactivación de microorganismos patógenos, lo que permite alargar su vida útil (Feria-Cuevas et al., 2019) sin cambios en los atributos sensoriales (Gandy et al., 2008); además, debe de cumplir con los criterios de calidad estipulados en dicha norma y sin la presencia de agentes adulterantes (Noa-Pérez et al., 2019).

En la actualidad, existe gran variedad de marcas de leche entera pasteurizada que se expenden en supermercados o tiendas de abarrotes (Noa-Pérez et al., 2019). Estas presumen en su etiqueta cumplir con lo establecido en la NOM-155SCFI-2012, y debido a que la leche es un producto que se ubica dentro de la canasta básica, es importante verificar y monitorear constantemente que las marcas cumplan con lo reportado en su etiquetado, así como lo estipulado en la normatividad vigente (Reyes et al., 2007). En el año 2006, el Consejo para el Fomento de la Calidad de Leche y sus Derivados (COFOCALEC) realizó un estudio a nivel nacional sobre el cumplimiento normativo de diversos productos lácteos (se incluía leche entera pasteurizada, $\mathrm{n}=154$ ) recolec- tados en puntos de venta, donde se reportó que el $79 \%$ ( $\mathrm{n}=$ 453) de los productos evaluados incumplían con las especificaciones mencionadas en la normatividad vigente, principalmente en el contenido de proteína, grasa, lactosa y sólidos totales, además del punto crioscópico y acidez titulable. Asimismo, las principales causas de incumplimiento en relación con los requisitos de información comercial fueron: información nutrimental, denominación comercial y contenido neto (COFOCALEC, 2006).

La NOM-051-SCFI/SSA1-2010 establece las características que debe contener el etiquetado de los alimentos y bebidas envasadas, con el objetivo de señalar las características del producto y evitar confundir o engañar a los compradores. Aunado a lo anterior, la NOM-091-SSA1-1994 menciona que la etiqueta de los productos lácteos, particularmente la leche pasteurizada de vaca debe señalar la fecha de caducidad con letra o número, el día, mes y año, así como incluir la leyenda "Manténgase en refrigeración" o "Consérvese en refrigeración”. En este contexto, la industria láctea con el objetivo de prolongar la vida útil de este producto ha hecho que la leche pase por diversos procesos antes de llegar a la mesa del consumidor. Algunos procesos se centran en asegurar la calidad sanitaria, mientras que otros están diseñados para disminuir los contenidos originales de proteína, grasa, lactosa u otros componentes. Sin embargo, cuando la leche pasteurizada envasada presenta valores de proteína por debajo de lo estipulado en la normatividad vigente, el producto resultante ya no puede seguir denominándose leche, sino fórmula láctea o producto lácteo combinado y debe mencionarse en la etiqueta (PROFECO, 2004).

Recientemente, se reportó la presencia de agentes adulterantes en el $68.5 \%$ de muestras de leche entera pasteurizada que se expenden en el estado de Jalisco (12 marcas evaluadas) (NoaPérez et al., 2019), principalmente aquellos relacionados con la inhibición del crecimiento microbiano (Noa-Pérez et al., 2021). Aunado a lo anterior, en el año 2020, la Procuraduría Federal del Consumidor prohibió la venta de 19 marcas de queso y 2 de yogurt por contener datos engañosos en su etiquetado e incumplir con la normatividad oficial vigente 
(Secretaría de Economía, 2020). Por lo que, el objetivo de este trabajo fue evaluar el contenido, información nutrimental y cumplimiento con la normatividad oficial mexicana de ocho marcas de leche entera pasteurizada que se expenden en supermercados y tiendas de abarrotes de la Ciudad de Tepatitlán de Morelos, Jalisco.

\section{Materiales y métodos}

Las muestras de leche se obtuvieron mediante un muestreo no probabilístico por conveniencia, donde se consideraron las ocho marcas de leche de mayor presencia en supermercados y tiendas de abarrotes del municipio de Tepatitlán de Morelos, Jalisco, México. Se compró leche entera pasteurizada en presentación de un litro, con fecha de caducidad vigente, condiciones de refrigeración usuales y sin alteraciones físicas aparentes. El muestreo se realizó durante tres días consecutivos asegurando que cada muestra fuese de un lote diferente. Se obtuvieron muestras de tres lotes diferentes y cada lote por duplicado. Además, se incluyó una muestra de leche cruda recién ordeñada (1 hora) de un productor local como comparativo de la calidad fisicoquímica de la leche entera pasteurizada, ya que de acuerdo con la definición de leche entera pasteurizada emitida por la PROFECO (2004), este producto comercial se asemeja al alimento original, y por norma debe contener al menos $30 \mathrm{~g} / \mathrm{L}$ de grasa butírica.

Las muestras fueron trasladadas conservando la cadena de frío, al Laboratorio de Análisis Fisicoquímicos de Alimentos del Departamento de Ingenierías del Centro Universitario de los Altos (CUAltos) de la Universidad de Guadalajara hasta su posterior análisis. Para el transporte de las muestras se siguieron las recomendaciones mencionadas en la NOM-109SSA1-1994.

Una vez en el laboratorio, se evaluó la información presente en la etiqueta con respecto a lo establecido en la NOM-051SCFI/SSA1-2010 (fecha de caducidad, leyenda "manténgase en refrigeración" y contenido nutrimental); y se verificó el contenido neto del producto utilizando una probeta graduada.

El contenido de proteína, caseína, grasa, lactosa, sólidos totales, sólidos no grasos, punto crioscópico y densidad se cuantificaron con un equipo MilkoScanTM (Foss, FT 120, tipo 71200, Hillerod, Dinamarca), el cual se calibró con una muestra certificada (DQCI, Set 1442) de acuerdo con la AOAC (1990). Previó al análisis de la muestra el equipo se acondicionó siguiendo las instrucciones del fabricante. La preparación de la muestra $(60 \mathrm{~mL})$ consistió en calentar la leche a $40{ }^{\circ} \mathrm{C}$ en un baño de agua, seguido de un enfriamiento a $20{ }^{\circ} \mathrm{C}$.

Por su parte, para la cuantificación de la acidez titulable se utilizó la metodología citada en la NOM-091-SSA1-1994. Adicionalmente, se determinó el pH (potenciómetro Hanna, pH 313), según lo establecido por la AOAC (2005; método 32.016).

Por último, los resultados de los análisis físico-químicos de las muestras fueron comparados con la información nutrimental reportada en la etiqueta y con las especificaciones de la NOM-155-SCFI-2012.

\section{Análisis estadístico de datos}

Los resultados se obtuvieron de tres experimentos independientes (3 lotes de leche, cada lote por duplicado, 8 marcas evaluadas) y cuantificados por duplicado $(n=48)$. Los resultados se expresaron como la media \pm desviación estándar. El análisis estadístico de los datos se dividió en varias secciones. Para la comparación entre marcas se aplicó una prueba de Kruskal-Wallis (debido a que no se cumplieron los supuestos de normalidad y homogeneidad de varianza; $p$ $<0.05$ ); acompañado de una comparación múltiple por rangos, mientras que el efecto del lote de producción por marca de leche se realizó mediante un análisis de varianza de una vía $(\alpha$ $=0.05)$ seguido de una prueba de Tukey $(p<0.05)$ para la comparación de medias. En lo referente a la comparación de los resultados con lo reportado en la etiqueta se utilizó una prueba T de Student $(p<0.05)$. Asimismo, se realizó una correlación de Pearson para identificar las relaciones entre las variables de estudio. Adicionalmente, se efectuó un análisis de clúster de jerarquías para determinar las relaciones entre las marcas de leche. Los análisis estadísticos se realizaron utilizando el paquete estadístico STATISTICA v.12 (Statsoft@, Tulsa, USA).

\section{Resultados y discusión}

\section{Información comercial en el etiquetado}

En el Cuadro 1 se presentan los datos sobre la información comercial de las distintas marcas de leche entera pasteurizada estudiadas. En general, los envases de leche no presentaban daños aparentes. Tal como puede apreciarse, todas las marcas de leche evaluadas cumplen con presentar la información comercial en su etiquetado, las cual incluye fecha de caducidad y lote de producción, leyenda "manténgase en refrigeración" o "consérvese en refrigeración" y contenido neto, tal como lo estipula la normatividad (NOM-091-SSA1-1994). Sin embargo, referente a la cuantificación del contenido neto de los productos, la marca G no cumplió con lo que menciona en su etiqueta $(1000 \mathrm{~mL})$ con un contenido neto promedio de $963 \pm 20$ $\mathrm{mL}$. El etiquetado en los alimentos es la principal fuente de información que el fabricante le proporciona al consumidor, por lo que, el no cumplir con lo ahí estipulado puede considerarse un engaño, con consecuente daño económico al consumidor (Tolentino-Mayo et al., 2018).

\begin{tabular}{|c|c|c|c|c|c|c|}
\hline Marca & Tipo de envase & $\begin{array}{l}\text { Información } \\
\text { sobre la } \\
\text { fecha de } \\
\text { caducidad }\end{array}$ & $\begin{array}{l}\text { Contiene } \\
\text { lote de } \\
\text { producción }\end{array}$ & $\begin{array}{c}\text { Presenta la } \\
\text { leyenda } \\
\text { "Consérvese en } \\
\text { refrigeración" }\end{array}$ & $\begin{array}{l}\text { Presenta } \\
\text { daños } \\
\text { aparentes }\end{array}$ & $\begin{array}{l}\text { Cumple con } \\
\text { el contenido } \\
\text { neto }\end{array}$ \\
\hline A & Garrafa de plástico & $\mathrm{Si}$ & $\mathrm{Si}$ & $\mathrm{Si}$ & No & $\mathrm{Si}$ \\
\hline B & Garrafa de plástico & $\mathrm{Si}$ & $\mathrm{Si}$ & $\mathrm{Si}$ & No & $\mathrm{Si}$ \\
\hline $\mathrm{C}$ & Garrafa de plástico & $\mathrm{Si}$ & $\mathrm{Si}$ & $\mathrm{Si}$ & No & $\mathrm{Si}$ \\
\hline D & Garrafa de plástico & $\mathrm{Si}$ & $\mathrm{Si}$ & $\mathrm{Si}$ & No & $\mathrm{Si}$ \\
\hline E & Tetrabrik & $\mathrm{Si}$ & $\mathrm{Si}$ & $\mathrm{Si}$ & No & $\mathrm{Si}$ \\
\hline $\mathrm{F}$ & Garrafa de plástico & $\mathrm{Si}$ & $\mathrm{Si}$ & $\mathrm{Si}$ & No & $\mathrm{Si}$ \\
\hline G & Bolsa pack & $\mathrm{Si}$ & $\mathrm{Si}$ & $\mathrm{Si}$ & No & No \\
\hline $\mathrm{H}$ & Garrafa de plástico & $\mathrm{Si}$ & $\mathrm{Si}$ & $\mathrm{Si}$ & No & $\mathrm{Si}$ \\
\hline
\end{tabular}


Composición físico-química de la leche

En el Cuadro 2 se presenta información acerca del contenido de proteína, caseína, grasa, lactosa y sólidos no grasos de la leche entera pasteurizada por marca y lote de producción. En lo referente a la composición química de la leche por lotes de la misma marca existen diferencias $(p<0.05)$ en todos los parámetros evaluados para las marcas $\mathrm{A}, \mathrm{B}, \mathrm{D}, \mathrm{F}, \mathrm{G}, \mathrm{y} \mathrm{H}$. Mientras que, la marca $\mathrm{C}$ solo presentó diferencias en el contenido de grasa $(\mathrm{p}<0.05)$, siendo la marca $\mathrm{C}$ la única que no mostró diferencias entre lotes $(\mathrm{p}>0.05)$. Según lo mencionado por Galván et al. (2005), durante el proceso de transformación de leche cruda en leche pasteurizada se llevan a cabo varios muestreos para determinar el contenido de proteína y grasa, posteriormente se somete a un proceso de homogenización y estandarización, en el cual se adiciona o retira grasa o proteína según sea el caso. La evidencia sugiere que los procesos de control de calidad de las empresas dedicadas a la venta de leche entera pasteurizada no son los correctos.
Respecto a la composición química de la leche, existen diferencias entre marcas $(p<0.05)$ para los parámetros evaluados (Cuadro 2). La marca A presentó los valores más bajos en todos los parámetros (proteína $21.95 \mathrm{~g} / \mathrm{L}$, caseína $12.73 \mathrm{~g} / \mathrm{L}$, grasa $17.90 \mathrm{~g} / \mathrm{L}$, lactosa $37.10 \mathrm{~g} / \mathrm{L}$ y sólidos no grasos $58.80 \mathrm{~g} / \mathrm{L})$, mientras que la marca $\mathrm{H}$ exhibió los valores más altos en proteína (28.56 g/L), caseína (20.91 g/L), lactosa $(47.64 \mathrm{~g} / \mathrm{L})$ y sólidos no grasos $(78.92 \mathrm{~g} / \mathrm{L})$. A su vez, la marca B mostró los valores más altos de grasa (28.86 g/L). De acuerdo con la PROFECO (2004), la leche entera pasteurizada es la más cercana a la leche cruda con respecto a su composición química. Sin embargo, al comparar la composición química de la leche pasteurizada con leche cruda, únicamente las marcas $\mathrm{E}$ y $\mathrm{H}$ presentaron semejanza en el contenido de proteína, caseína, lactosa y sólidos no grasos. Cabe destacar que todas las marcas presentaron valores por debajo de lo estipulado en la normatividad oficial vigente para leche pasteurizada (NOM-151-SCFI-2012) en la mayoría de los parámetros evaluados, excepto para lactosa. Estos resultados

Cuadro 2. Composición química de leche por marca y lote de producción.

\begin{tabular}{|c|c|c|c|c|c|c|}
\hline Marca & Lote & $\begin{array}{c}\text { Proteína } \\
(\mathrm{g} / \mathrm{L})\end{array}$ & $\begin{array}{c}\text { Caseina } \\
(\mathrm{g} / \mathrm{L})\end{array}$ & $\begin{array}{l}\text { Grasa } \\
(\mathrm{g} / \mathrm{L})\end{array}$ & $\begin{array}{c}\text { Lactosa } \\
(\mathrm{g} / \mathrm{L})\end{array}$ & $\begin{array}{l}\text { SNG } \\
(\mathrm{g} / \mathrm{L})\end{array}$ \\
\hline \multirow{3}{*}{ A } & 1 & $17.23 \pm 1.52 \mathrm{~B}$ & $7.23 \pm 2.35 \mathrm{~B}$ & $18.53 \pm 2.25 B$ & $31.16 \pm 2.75 \mathrm{~B}$ & $46.10 \pm 5.30 \mathrm{~B}$ \\
\hline & 2 & $23.36 \pm 0.65 \mathrm{~A}$ & $14.20 \pm 0.80 \mathrm{~A}$ & $25.70 \pm 0.80 \mathrm{~A}$ & $38.73 \pm 0.95 \mathrm{~A}$ & $62.26 \pm 1.95 \mathrm{~A}$ \\
\hline & 3 & $25.26 \pm 0.25 \mathrm{~A}$ & $16.76 \pm 0.45 \mathrm{~A}$ & $27.26 \pm 0.15 \mathrm{~A}$ & $41.60 \pm 0.50 \mathrm{~A}$ & $68.30 \pm 1.00 \mathrm{~A}$ \\
\hline Promedio A & & $21.95 \pm 3.75^{d}$ & $12.73 \pm 4.54^{d}$ & $23.83 \pm 4.20^{d}$ & $37.10 \pm 4.89^{d}$ & $58.88 \pm 10.34^{d}$ \\
\hline \multirow{3}{*}{ B } & 1 & $25.10 \pm 0.20 \mathrm{~B}$ & $16.96 \pm 0.05 \mathrm{~B}$ & $28.00 \pm 0.30 \mathrm{~B}$ & $42.23 \pm 0.05 B$ & $69.13 \pm 0.15 B$ \\
\hline & 2 & $26.10 \pm 0.50 \mathrm{~A}$ & $17.93 \pm 0.25 \mathrm{~A}$ & $29.13 \pm 0.25 \mathrm{~A}$ & $43.40 \pm 0.10 \mathrm{~A}$ & $71.46 \pm 0.55 \mathrm{~A}$ \\
\hline & 3 & $26.36 \pm 0.35 \mathrm{~A}$ & $18.16 \pm 0.25 \mathrm{~A}$ & $29.46 \pm 0.35 \mathrm{~A}$ & $43.80 \pm 0.40 \mathrm{~A}$ & $72.20 \pm 0.80 \mathrm{~A}$ \\
\hline Promedio B & & $25.85 \pm 0.66^{c}$ & $17.68 \pm 0.57^{c}$ & $28.86 \pm 0.71^{b}$ & $43.14 \pm 0.73^{c}$ & $70.93 \pm 1.47^{c}$ \\
\hline \multirow{3}{*}{$\mathrm{C}$} & 1 & $26.86 \pm 0.25 \mathrm{~A}$ & $18.60 \pm 0.01 \mathrm{~A}$ & $28.06 \pm 0.25 \mathrm{~A}$ & $44.50 \pm 0.30 \mathrm{~A}$ & $73.53 \pm 0.25 \mathrm{~A}$ \\
\hline & 2 & $2.6 .70 \pm 0.50 \mathrm{~A}$ & $18.66 \pm 0.35 \mathrm{~A}$ & $28.36 \pm 0.55 \mathrm{~A}$ & $44.36 \pm 0.35 \mathrm{~A}$ & $73.23 \pm 0.65 \mathrm{~A}$ \\
\hline & 3 & $27.00 \pm 0.10 \mathrm{~A}$ & $18.96 \pm 0.15 \mathrm{~A}$ & $28.36 \pm 0.05 \mathrm{~A}$ & $44.80 \pm 0.20 \mathrm{~A}$ & $74.10 \pm 0.20 \mathrm{~A}$ \\
\hline Promedio C & & $26.85 \pm 0.31^{\text {ac }}$ & $18.74 \pm 0.25^{\mathrm{bc}}$ & $28.26 \pm 0.33^{\mathrm{bc}}$ & $44.55 \pm 0.31^{\mathrm{bc}}$ & $76.62 \pm 0.52^{\mathrm{bc}}$ \\
\hline \multirow{3}{*}{$\mathrm{D}$} & 1 & $28.20 \pm 0.30 \mathrm{~B}$ & $19.80 \pm 0.30 \mathrm{~B}$ & $20.30 \pm 1.90 \mathrm{~A}$ & $46.40 \pm 0.40 \mathrm{~B}$ & $77.63 \pm 1.05 \mathrm{~B}$ \\
\hline & 2 & $28.66 \pm 0.15 \mathrm{AB}$ & $20.30 \pm 0.01 \mathrm{~A}$ & $17.06 \pm 0.05 B$ & $47.10 \pm 0.01 \mathrm{~A}$ & $78.90=0.30 \mathrm{AB}$ \\
\hline & 3 & $28.80 \pm 0.10 \mathrm{~B}$ & $20.50 \pm 0.10 \mathrm{~A}$ & $16.33 \pm 0.15 B$ & $47.33 \pm 0.15 \mathrm{~A}$ & $79.43 \pm 0.45 \mathrm{~A}$ \\
\hline Promedio D & & $28.55 \pm 0.32^{\mathrm{ab}}$ & $20.20 \pm 0.35^{b}$ & $17.90 \pm 2.06^{\mathrm{e}}$ & $46.94 \pm 0.47^{\mathrm{ab}}$ & $78.65 \pm 0.99^{\mathrm{ab}}$ \\
\hline \multirow{3}{*}{$\mathrm{E}$} & 1 & $28.40 \pm 0.10 \mathrm{~A}$ & $20.56 \pm 0.15 \mathrm{~A}$ & $23.16 \pm 1.75 B$ & $47.06 \pm 0.15 \mathrm{~A}$ & $78.30 \pm 0.30 \mathrm{~A}$ \\
\hline & 2 & $28.46 \pm 0.25 \mathrm{~A}$ & $20.80 \pm 0.10 \mathrm{~A}$ & $26.30 \pm 0.40 \mathrm{~A}$ & $47.20 \pm 0.10 \mathrm{~A}$ & $78.66 \pm 0.25 \mathrm{~A}$ \\
\hline & 3 & $28.46 \pm 0.05 \mathrm{~A}$ & $20.80 \pm 0.01 \mathrm{~A}$ & $27.20 \pm 0.10 \mathrm{~A}$ & $47.36 \pm 0.15 \mathrm{~A}$ & $78.56 \pm 0.05 \mathrm{~A}$ \\
\hline Promedio E & & $28.44 \pm 0.14^{\mathrm{ab}}$ & $20.72 \pm 0.14^{\mathrm{ab}}$ & $25.55 \pm 2.04^{c d}$ & $47.21 \pm 0.17^{\mathrm{a}}$ & $78.51 \pm 0.25^{\mathrm{ab}}$ \\
\hline \multirow{3}{*}{$\mathrm{F}$} & 1 & $27.33 \pm 0.25 B$ & $19.60 \pm 0.30 \mathrm{~B}$ & $27.36 \pm 0.15 \mathrm{~A}$ & $46.33 \pm 0.25 B$ & $76.30 \pm 0.70 \mathrm{~B}$ \\
\hline & 2 & $26.96 \pm 0.05 B$ & $19.20 \pm 0.01 B$ & $27.66 \pm 0.05 \mathrm{~A}$ & $45.90 \pm 0.10 \mathrm{~B}$ & $75.26 \pm 0.15 B$ \\
\hline & 3 & $28.80 \pm 0.40 \mathrm{~A}$ & $20.93 \pm 0.45 \mathrm{~A}$ & $27.80 \pm 0.01 \mathrm{~A}$ & $47.10 \pm 0.30 \mathrm{~A}$ & $78.80 \pm 0.90 \mathrm{~A}$ \\
\hline Promedio F & & $27.70 \pm 0.87 \mathrm{ac}$ & $19.91 \pm 0.83^{\mathrm{bc}}$ & $27.61 \pm 0.20^{\mathrm{kc}}$ & $46.44 \pm 0.56^{\mathrm{ab}}$ & $76.78 \pm 1.65^{\mathrm{ab}}$ \\
\hline \multirow{3}{*}{$\mathrm{G}$} & 1 & $27.73 \pm 0.45 \mathrm{~A}$ & $19.83 \pm 0.35 \mathrm{~B}$ & $25.90 \pm 0.40 \mathrm{~A}$ & $47.03 \pm 0.15 \mathrm{~A}$ & $76.76 \pm 0.55 \mathrm{~B}$ \\
\hline & 2 & $27.83 \pm 0.25 \mathrm{~A}$ & $20.06 \pm 0.25 \mathrm{AB}$ & $26.23 \pm 0.25 \mathrm{~A}$ & $47.50 \pm 0.10 \mathrm{~A}$ & $77.60 \pm 0.50 \mathrm{AB}$ \\
\hline & 3 & $28.16 \pm 0.05 \mathrm{~A}$ & $20.46 \pm 0.05 \mathrm{~A}$ & $26.53 \pm 0.05 \mathrm{~A}$ & $47.63 \pm 0.05 \mathrm{~A}$ & $78.40 \pm 0.10 \mathrm{~A}$ \\
\hline Promedio G & & $27.91 \pm 0.32^{b}$ & $20.12 \pm 0.35^{b}$ & $26.22 \pm 0.36^{\mathrm{bcd}}$ & $47.38 \pm 0.28^{a}$ & $77.58 \pm 0.80^{\mathrm{ab}}$ \\
\hline \multirow{3}{*}{$\mathrm{H}$} & 1 & $28.46 \pm 0.15 \mathrm{~A}$ & $20.80 \pm 0.10 \mathrm{~B}$ & $27.60 \pm 0.20 \mathrm{~B}$ & $47.63 \pm 0.57 \mathrm{AB}$ & $78.70 \pm 0.10 \mathrm{~B}$ \\
\hline & 2 & $28.60 \pm 0.01 \mathrm{~A}$ & $20.93 \pm 0.05 \mathrm{AB}$ & $27.90 \pm 0.10 \mathrm{AB}$ & $47.56 \pm 0.05 B$ & $78.96 \pm 0.05 \mathrm{~A}$ \\
\hline & 3 & $28.63 \pm 0.05 \mathrm{~A}$ & $21.00 \pm 0.01 \mathrm{~A}$ & $28.10 \pm 0.10 \mathrm{~A}$ & $47.73 \pm 0.05 \mathrm{~A}$ & $79.10 \pm 0.10 \mathrm{~A}$ \\
\hline Promedio $\mathrm{H}$ & & $28.56 \pm 0.11^{\mathrm{ab}}$ & $20.91 \pm 0.10^{\mathrm{ab}}$ & $27.86 \pm 0.25 \mathrm{bc}$ & $47.64 \pm 0.08^{\mathrm{a}}$ & $78.92 \pm 0.19^{\mathrm{ab}}$ \\
\hline Control & & $30.45 \pm 1.34^{a}$ & $22.76 \pm 0.86^{a}$ & $34.15 \pm 2.91^{\mathrm{a}}$ & $47.45 \pm 0.45^{\mathrm{a}}$ & $81.86 \pm 2.29^{\mathrm{a}}$ \\
\hline Normatividad & & $\geq 30$ & $\geq 24$ & $\geq 30$ & $43-54$ & $\geq 83$ \\
\hline
\end{tabular}

Los valores son la media \pm desviación estándar. Letras mayúsculas diferentes por columna indican diferencias estadísticas significativas $(p<0.05)$ entre lotes de producción de la misma marca. Letras minúsculas por columna indican diferencias estadisticas significativas $(p<0.05)$ entre marcas de leche. SNG: sólidos no grasos. 
coinciden con lo reportado por Juárez-Barrientos et al. (2015), quienes informaron diferencias $(p<0.05)$ en la composición química (proteína, grasa, lactosa y sólidos no grasos) de ocho marcas de leche entera pasteurizada que se comercializa en Tuxtepec, Oaxaca, México, además de que el contenido de proteína (23 a $28.10 \mathrm{~g} / \mathrm{L}$ ), lactosa (34.40 a $39.90 \mathrm{~g} / \mathrm{L}$ ) y sólidos no grasos (70.33 a $81.30 \mathrm{~g} / \mathrm{L})$ no cumplían con lo estipulado en la normatividad oficial. Del mismo modo, Briñez et al. (2002) informaron diferencias significativas en los componentes nutricionales de cinco marcas de leche entera pasteurizada comercializada en Maracaibo, Venezuela. Por su parte, Awan et al. (2014) reporto diferencias $(p<0.05)$ en el contenido de grasa de ocho marcas que se expenden en mercados de Multan, Pakistán. Estos resultados pudieran estar relacionados con la calidad de la leche cruda (Juárez-Barrientos et al., 2015). Sin embargo, considerando el proceso de homogenización y estandarización que recibe la leche comercial previo a la pasteurización, estos resultados pudieran ser efecto directo del deficiente control de calidad en las empresas destinadas a la venta de leche pasteurizada (Galván et al., 2005).

En el Cuadro 3 se presenta información acerca de los parámetros fisicoquímicos $(\mathrm{pH}$, acidez titulable, punto crioscópico y densidad) de la leche entera pasteurizada por marca y lote de producción. En general, existen diferencias $(\mathrm{p}<$ 0.05) en los parámetros fisicoquímicos entre los lotes de las distintas marcas, excepto para las marcas $\mathrm{C}$ y E que fueron las únicas que no presentaron variación entre lotes $(p>0.05)$.

Cuadro 3. Parámetros fisicoquímicos de las distintas marcas y lotes de producción de leche entera pasteurizada.

\begin{tabular}{|c|c|c|c|c|c|}
\hline Marca & Lote & $\mathrm{pH}$ & $\begin{array}{c}\text { Acidez titulable } \\
\text { (g ácido láctico/L) }\end{array}$ & $\begin{array}{c}\text { Punto crioscópico } \\
\left({ }^{\circ} \mathrm{C}\right)\end{array}$ & $\begin{array}{c}\text { Densidad } \\
(\mathrm{g} / \mathrm{mL})\end{array}$ \\
\hline \multirow{3}{*}{ A } & 1 & $6.64 \pm 0.03 \mathrm{~A}$ & $2.02 \pm 0.06 \mathrm{~A}$ & $-0.285 \pm 0.034 \mathrm{~B}$ & $1.016 \pm 0.002 B$ \\
\hline & 2 & $6.67 \pm 0.01 \mathrm{~A}$ & $1.77 \pm 0.02 B$ & $-0.390 \pm 0.012 \mathrm{~A}$ & $1.022 \pm 0.001 \mathrm{~A}$ \\
\hline & 3 & $6.68 \pm 0.01 \mathrm{~A}$ & $1.86 \pm 0.11 \mathrm{~B}$ & $-0.422 \pm 0.003 \mathrm{~A}$ & $1.024 \pm 0.005 \mathrm{~A}$ \\
\hline Promedio A & & $6.66 \pm 0.02^{\mathrm{d}}$ & $1.89 \pm 0.13^{b}$ & $-0.365 \pm 0.064^{\mathrm{d}}$ & $1.020 \pm 0.004^{d}$ \\
\hline \multirow{3}{*}{ B } & 1 & $6.67 \pm 0.01 \mathrm{~A}$ & $1.83 \pm 0.04 \mathrm{~A}$ & $-0.430 \pm 0.002 \mathrm{~A}$ & $1.025 \pm 0.001 \mathrm{~B}$ \\
\hline & 2 & $6.67 \pm 0.01 \mathrm{~A}$ & $1.76 \pm 0.05 \mathrm{~A}$ & $-0.448 \pm 0.007 \mathrm{~A}$ & $1.026 \pm 0.001 \mathrm{~A}$ \\
\hline & 3 & $6.66 \pm 0.01 \mathrm{~A}$ & $1.77 \pm 0.02 \mathrm{~A}$ & $-0.455 \pm 0.007 \mathrm{~A}$ & $1.026 \pm 0.005 \mathrm{~A}$ \\
\hline Promedio B & & $6.70 \pm 0.04 \mathrm{~cd}$ & $1.79 \pm 0.05^{b}$ & $-0.444 \pm 0.012^{c}$ & $1.025 \pm 0.006^{c}$ \\
\hline \multirow{3}{*}{$\mathrm{C}$} & 1 & $6.79 \pm 0.01 \mathrm{~A}$ & $1.76 \pm 0.02 \mathrm{~A}$ & $-0.460 \pm 0.001 \mathrm{~A}$ & $1.027 \pm 0.001 \mathrm{~A}$ \\
\hline & 2 & $6.77 \pm 0.01 \mathrm{~A}$ & $1.79 \pm 0.04 \mathrm{~A}$ & $-0.457 \pm 0.008 \mathrm{~A}$ & $1.027 \pm 0.001 \mathrm{~A}$ \\
\hline & 3 & $6.77 \pm 0.01 \mathrm{~A}$ & $1.71 \pm 0.02 \mathrm{~A}$ & $-0.458 \pm 0.002 \mathrm{~A}$ & $1.027 \pm 0.005 \mathrm{~A}$ \\
\hline Promedio C & & $6.78 \pm 0.01^{b}$ & $1.75 \pm 0.04^{b}$ & $-0.458 \pm 0.004^{b c}$ & $1.027 \pm 0.004^{b c}$ \\
\hline \multirow{3}{*}{ D } & 1 & $6.73 \pm 0.01 B$ & $1.71 \pm 0.02 \mathrm{~B}$ & $-0.482 \pm 0.007^{2}$ & $1.029 \pm 0.005 \mathrm{~A}$ \\
\hline & 2 & $6.74 \pm 0.01 B$ & $1.52 \pm 0.04 B$ & $-0.489 \pm 0.004 \mathrm{~A}$ & $1.031 \pm 0.001 \mathrm{~B}$ \\
\hline & 3 & $6.77 \pm 0.01 \mathrm{~A}$ & $1.47 \pm 0.04 \mathrm{~A}$ & $-0.488 \pm 0.002 \mathrm{~A}$ & $1.031 \pm 0.001 \mathrm{~B}$ \\
\hline Promedio D & & $6.74 \pm 0.02^{b c}$ & $1.57 \pm 0.11^{\mathrm{c}}$ & $-0.486 \pm 0.005^{\mathrm{ab}}$ & $1.030 \pm 0.007^{2}$ \\
\hline \multirow{3}{*}{ E } & 1 & $6.78 \pm 0.01 \mathrm{~A}$ & $1.50 \pm 0.02 \mathrm{~A}$ & $-0.483 \pm 0.003 \mathrm{~A}$ & $1.030 \pm 0.001 \mathrm{~A}$ \\
\hline & 2 & $6.78 \pm 0.01 \mathrm{~A}$ & $1.46 \pm 0.02 \mathrm{~A}$ & $-0.486 \pm 0.006 \mathrm{~A}$ & $1.030 \pm 0.001 \mathrm{~A}$ \\
\hline & 3 & $6.77 \pm 0.01 \mathrm{~A}$ & $1.47 \pm 0.04 \mathrm{~A}$ & $-0.487 \pm 0.001 \mathrm{~A}$ & $1.030 \pm 0.001 \mathrm{~A}$ \\
\hline Promedio E & & $6.78 \pm 0.01^{b}$ & $1.48 \pm 0.03^{c}$ & $-0.485 \pm 0.003^{a b}$ & $1.030 \pm 0.001^{\mathrm{a}}$ \\
\hline \multirow{3}{*}{$\mathrm{F}$} & 1 & $6.86 \pm 0.01 B$ & $1.25 \pm 0.04 \mathrm{~A}$ & $-0.472 \pm 0.001 \mathrm{~B}$ & $1.029 \pm 0.001 \mathrm{~A}$ \\
\hline & 2 & $6.85 \pm 0.01 B$ & $1.29 \pm 0.04 \mathrm{AB}$ & $-0.468 \pm 0.001 B$ & $1.028 \pm 0.001 B$ \\
\hline & 3 & $6.82 \pm 0.01 \mathrm{~A}$ & $1.38 \pm 0.04 B$ & $-0.490 \pm 0.006 \mathrm{~A}$ & $1.029 \pm 0.005 \mathrm{~A}$ \\
\hline Promedio F & & $6.84 \pm 0.01^{\mathrm{a}}$ & $1.31 \pm 0.07^{d}$ & $-0.476 \pm 0.010^{2 b c}$ & $1.028 \pm 0.007^{\mathrm{ab}}$ \\
\hline \multirow{3}{*}{ G } & 1 & $6.79 \pm 0.01 \mathrm{~A}$ & $1.40 \pm 0.02 B$ & $-0.496 \pm 0.001 \mathrm{~A}$ & $1.029 \pm 0.001 \mathrm{~A}$ \\
\hline & 2 & $6.73 \pm 0.01 B$ & $1.56 \pm 0.04 \mathrm{~A}$ & $-0.494 \pm 0.003 \mathrm{~A}$ & $1.029 \pm 0.005 \mathrm{~A}$ \\
\hline & 3 & $6.72 \pm 0.01 B$ & $1.50 \pm 0.02 \mathrm{~A}$ & $-0.496 \pm 0.003 \mathrm{~A}$ & $1.030 \pm 0.001 \mathrm{~A}$ \\
\hline Promedio $\mathrm{G}$ & & $6.74 \pm 0.03^{b c}$ & $1.49 \pm 0.07^{c}$ & $-0.495 \pm 0.002^{\mathrm{a}}$ & $1.029 \pm 0.005^{2}$ \\
\hline \multirow{3}{*}{$\mathrm{H}$} & 1 & $6.77 \pm 0.01 \mathrm{~A}$ & $1.55 \pm 0.02 \mathrm{~A}$ & $-0.495 \pm 0.001 \mathrm{~A}$ & $1.030 \pm 0.001 \mathrm{~A}$ \\
\hline & 2 & $6.76 \pm 0.01 \mathrm{~A}$ & $1.44 \pm 0.06 \mathrm{~B}$ & $-0.494 \pm 0.001 \mathrm{~A}$ & $1.030 \pm 0.001 \mathrm{~A}$ \\
\hline & 3 & $6.77 \pm 0.01 \mathrm{~A}$ & $1.44 \pm 0.06 \mathrm{~B}$ & $-0.497 \pm 0.001 \mathrm{~A}$ & $1.030 \pm 0.001 \mathrm{~A}$ \\
\hline Promedio $\mathrm{H}$ & & $6.77 \pm 0.01^{b}$ & $1.48 \pm 0.07^{c}$ & $-0.494 \pm 0.001^{\mathrm{a}}$ & $1.030 \pm 0.001^{2}$ \\
\hline Control & & $6.60 \pm 0.08^{e}$ & $2.27 \pm 0.19^{\mathrm{a}}$ & $-0.512 \pm 0.008^{\mathrm{a}}$ & $1.029 \pm 0.008^{\mathrm{ab}}$ \\
\hline Normatividad & & & $1.3-1.7$ & -0.530 a -0.560 & $\geq 1.029$ \\
\hline
\end{tabular}


En general, los valores de $\mathrm{pH}$ se ubicaron en un rango de 6.66 a 6.84 dependiendo de la marca. Negri (2005) menciona que el proceso de pasteurización puede causar cambios en el $\mathrm{pH}$ de la leche, asociados a la pérdida de $\mathrm{CO}_{2}$ y a la precipitación del fosfato de calcio.

Por su parte, los valores de acidez titulable se encuentran en un rango de 1.48 a $1.89 \mathrm{~g}$ de ácido láctico/L $(p<0.05)$. La marca A y B son las que presentan mayor contenido de acidez (1.89 y $1.79 \mathrm{~g}$ de ácido láctico/L, respectivamente), los cuales se encuentran por encima de lo estipulado en la normatividad oficial (1.3 - $1.7 \mathrm{~g}$ ácido láctico/L). Noa-Pérez et al. (2019) reportaron que el $92.2 \%$ de las muestras de leche pasteurizada (12 marcas) que se vende en la ciudad de Guadalajara cumplieron con la normatividad oficial para este parámetro. Los autores mencionan que valores de acidez $>1.7 \mathrm{~g} / \mathrm{L}$ se asocian al rompimiento de la cadena de frío durante el transporte y almacenamiento de la leche, lo que propicia el crecimiento de bacterias ácido lácticas $\mathrm{u}$ otros microorganismos deterioradores del producto. En contraparte, Juárez-Barrientos et al. (2015) reportaron que algunas marcas (7 de 8 ) de leche entera pasteurizada que se expenden en Tuxtepec, Oaxaca presentaban valores $(0.9$ a $1.1 \mathrm{~g}$ ácido láctico/L) por debajo de lo especificado en la normatividad. Rondón et al. (2003) reportaron la presencia de sustancias neutralizadoras de ácido láctico en leche entera pasteurizada que se comercializa en el estado de Mérida en Venezuela, mismas que son añadidas con el fin de enmascarar los efectos asociados a una carga microbiana elevada.

En lo referente al punto crioscópico de la leche, los valores rondan entre -0.365 y $-0.495{ }^{\circ} \mathrm{C}$, con diferencias entre marcas $(p<0.05)$ (Cuadro 3). La marca A presentó los valores más bajos en este parámetro $\left(-0.365^{\circ} \mathrm{C}\right)$, mientras que los valores del resto de las marcas oscilaron entre -0.444 y $-0.495{ }^{\circ} \mathrm{C}$, valores por debajo de lo especificado en la normatividad oficial mexicana para leche entera pasteurizada (-0.530 a $\left.-0.560{ }^{\circ} \mathrm{C}\right)$. Tendencias similares se reportaron en los valores de crioscopia $\left(-0.400\right.$ a $\left.-0.470{ }^{\circ} \mathrm{C}\right)$ en ocho marcas comerciales de leche entera pasteurizada que se expenden en Tuxtepec, Oaxaca (Juárez-Barrientos et al., 2015). Asimismo, el 27\% (n = 141) de muestras de leche pasteurizada que se comercializa en la ciudad de Guadalajara, presentó valores por debajo del rango aceptable (-0.530 a $\left.-0.560{ }^{\circ} \mathrm{C}\right)$ (Noa-Pérez et al., 2019). Juárez-Barrientos et al. (2015) mencionan que valores fuera de norma del punto de congelación de la leche tratada térmicamente pudieran asociarse con la adición de agua, ya sea intencional o por condensación del agua dentro del pasteurizador durante el procesamiento.

Asimismo, la densidad de la leche en las marcas D, E, F, G y $\mathrm{H}$ presentan valores dentro de los rangos establecidos por la NOM-151-CSFI-2012 (1.029-1.031 g/mL) y similares a lo reportado en leche tratada térmicamente $(1.031 \mathrm{~g} / \mathrm{mL})$ (Bermúdez-Aguirre et al., 2009). Mientras que, las marcas A $(1.020 \mathrm{~g} / \mathrm{mL}), \mathrm{B}(1.025 \mathrm{~g} / \mathrm{mL})$ y C $(1.027 \mathrm{~g} / \mathrm{mL})$ mostraron valores por debajo de los rangos aceptables para leche pasteurizada. Juaréz-Barrientos et al. (2015) reportaron valores de densidad entre 1.024 y $1.028 \mathrm{~g} / \mathrm{mL}$ en ocho marcas comerciales de leche entera pasteurizada en Tuxtepec, Oaxaca. De acuerdo con Noa-Pérez et al. (2019) una práctica común para enmascarar el efecto de la adición de agua en leche y para aumentar la densidad de la misma, es la adición de sacarosa. Adicionalmente, Reyes et al. (2007) reportaron la presencia de suero de quesería en ocho marcas comerciales de leche entera pasteurizada que se expende en la ciudad de Aguascalientes, Aguascalientes, México (de las cuales tres son incluidas en este estudio), lo que influye directamente en su calidad fisicoquímica.

\section{Correlación entre parámetros de calidad de la leche}

En el Cuadro 4 se presenta el análisis de correlación de Pearson, el cual se basa en la correlación estadística de las variables de estudio. El contenido de proteína presentó correlación positiva con el contenido de lactosa $(r=0.957)$, sólidos no grasos (SNG) $(r=0.991)$, punto crioscópico $(r=$ $0.958)$ y densidad ( $r=0.936)$, mientras que la lactosa presentó correlación positiva con los SNG $(r=0.986)$, punto crioscópico $(r=0.997)$ y densidad $(r=0.988)$. Del mismo modo, los SNG presentaron correlación positiva con el punto crioscópico $(r=$ 0.985) y la densidad $(r=0.970)$, además de que el punto crioscópico presentó correlación positiva con la densidad ( $r=$ 0.980). La correlación sugiere que el aumento o decremento de cualquier componente (proteína, lactosa y grasa) de la leche tendrá impacto directo en el contenido de SNG, punto de congelación y densidad de la leche, lo que evidenciaría algún tipo de adulteración (Briñez et al., 2002). Comportamiento similar fue reportado por Juárez-Barrientos et al. (2015) en leche entera pasteurizada comercial, quienes informaron correlaciones entre contenido de proteína con lactosa $(r=0.90)$, SNG ( $r=0.85)$, punto crioscópico $(r=-0.93)$ y densidad $(r=$ 0.89 ), a su vez, la lactosa se correlacionó positivamente con los SNG $(\mathrm{r}=0.82)$, punto crioscópico $(r=-0.90)$ y densidad $(r=$ $0.92)$, mientras que el punto crioscópico se correlaciono con la densidad $(r=-0.96)$.

\begin{tabular}{|c|c|c|c|c|c|c|c|}
\hline & Proteina & Grasa & Lactosa & SNG & AT & PC & Densidad \\
\hline Proteina & 1.00 & $\begin{array}{c}0.290 \\
(p=0.449)\end{array}$ & $\begin{array}{c}0.957 \\
(p<0.001)\end{array}$ & $\begin{array}{c}0.991 \\
(p<0.001)\end{array}$ & $\begin{array}{c}-0.0534 \\
(p=0.891)\end{array}$ & $\begin{array}{c}0.958 \\
(p<0.001)\end{array}$ & $\begin{array}{c}0.936 \\
(p<0.001)\end{array}$ \\
\hline Grasa & & 1.00 & $\begin{array}{c}0.615 \\
(p=0.672)\end{array}$ & $\begin{array}{c}0.231 \\
(p=0.550)\end{array}$ & $\begin{array}{c}0.484 \\
(p=0.187)\end{array}$ & $\begin{array}{c}0.188 \\
(p=0.628)\end{array}$ & $\begin{array}{c}0.0179 \\
p=0.964\end{array}$ \\
\hline Lactosa & & & 1.00 & $\begin{array}{c}0.986 \\
(p<0.001)\end{array}$ & $\begin{array}{c}-0.319 \\
(p=0.403)\end{array}$ & $\begin{array}{c}0.997 \\
(p<0.001)\end{array}$ & $\begin{array}{c}0.988 \\
(p<0.001)\end{array}$ \\
\hline SNG & & & & 1.00 & $\begin{array}{c}-0.177 \\
(p=0.648)\end{array}$ & $\begin{array}{c}0.985 \\
(p<0.001)\end{array}$ & $\begin{array}{c}0.970 \\
(p<0.001)\end{array}$ \\
\hline AT & & & & & 1.00 & $\begin{array}{c}-0.285 \\
(p=0.457)\end{array}$ & $\begin{array}{c}-0.375 \\
(p=0.321)\end{array}$ \\
\hline PC & & & & & & 1.00 & $\begin{array}{c}0.980 \\
(p<0.001)\end{array}$ \\
\hline$\frac{\text { Densidad }}{{ }^{*} \mathrm{El} \text { par de }}$ & & & & 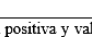 & & 0.05 & $\frac{1.00}{\text { n a increme }}$ \\
\hline
\end{tabular}

Cumplimiento del contenido nutrimental de leche entera pasteurizada con respecto al etiquetado

En el Cuadro 5 se presenta una comparación del contenido nutrimental (experimental) de la leche entera pasteurizada con lo reportado en su etiqueta. Tal como puede apreciarse, ninguna de las marcas de leche incluidas en este estudio cumple con el contenido nutrimental (proteína, grasa y lactosa) que se reporta 
en su etiquetado $(p<0.05)$; excepto por la marca $\mathrm{G}$ que solo cumple en el contenido de lactosa $(p>0.05)$. Arriaga et al. (2019) reportaron que el yogurt natural y estilo griego que se expende en la ciudad de Pachuca de Soto, Hidalgo, México cumplían con el contenido nutrimental informado en su etiquetado. Calderón et al. (2007) mencionan que en las empresas procesadoras de productos lácteos, una práctica común es la mezcla de leches de diferentes procedencias, por tal motivo se debe realizar una corrección de los componentes y tener extremo control de calidad en cuanto a la concentración reportada en el etiquetado. Tolentino-Mayo et al. (2018) sugieren que las instancias reguladoras deben de implementar un sistema de evaluación que garantice el cumplimiento de lo expuesto en el etiquetado de los alimentos. De acuerdo con la Fundación Vasca para la seguridad agroalimentaria Elika (2011) el etiquetado correcto de los alimentos es fundamental para el control del fraude alimentario. Si bien es cierto, este tipo de fraude no pone en riesgo la salud del consumidor, la adquisición y consumo de un producto alimenticio debe ser una decisión soberana del consumidor y no un engaño del fabricante debido a que la etiqueta no ostenta el contenido nutrimental real del producto (Reyes et al., 2007).

Comparación del contenido nutrimental de leche entera pasteurizada con respecto a la normatividad oficial mexicana Dentro de la legislación mexicana existe una norma aplicable para leche pasteurizada (NOM-155-SCFI-2012) en la cual se hace referencia a las especificaciones fisicoquímicas y nutrimentales (mínimos y máximos) que deben de cumplir los productos lácteos comerciales sujetos a la misma. En el Cuadro 6 , se presenta el porcentaje de cumplimiento de las distintas marcas comerciales de leche entera pasteurizada con respecto a las especificaciones de la NOM-155-SCFI-2012. Considerando las ocho especificaciones que marca la normatividad deben de cumplir las marcas de leche comerciales $(100 \%$ de cumplimiento), las marcas que presentan mayor cumplimiento son D, E, F, G y H (37.5\%), seguido de la marca B (25\%) y por

Cuadro 5. Comparación de los valores experimentales contra valores en etiqueta.

\begin{tabular}{cccccc}
\hline Marca & Parámetro & $\begin{array}{c}\text { Valor experimental } \\
(\mathrm{g} / \mathrm{L})\end{array}$ & $\begin{array}{c}\text { Valor en etiqueta } \\
(\mathrm{g} / \mathrm{L})\end{array}$ & $\begin{array}{c}\text { Error } \\
(\%)\end{array}$ & Valor- $p$ \\
\hline \multirow{4}{*}{$\mathrm{A}$} & Proteína & $21.95 \pm 3.75$ & 31.2 & 29.64 & $<0.0001$ \\
& Grasa & $23.83 \pm 4.20$ & 33.2 & 28.22 & 0.0003 \\
& Lactosa & $37.10 \pm 4.89$ & 48 & 22.70 & 0.0003 \\
\hline \multirow{4}{*}{ B } & Proteína & $25.85 \pm 0.66$ & 31 & 16.61 & $<0.0001$ \\
& Grasa & $28.86 \pm 0.71$ & 33 & 13.33 & $<0.0001$ \\
& Lactosa & $43.14 \pm 0.73$ & 48 & 10.12 & $<0.0001$ \\
\hline \multirow{4}{*}{ C } & Proteína & $26.85 \pm 0.31$ & 31 & 13.38 & $<0.0001$ \\
& Grasa & $28.26 \pm 0.33$ & 30 & 5.80 & $<0.0001$ \\
& Lactosa & $44.55 \pm 0.31$ & 50 & 10.90 & $<0.0001$ \\
\hline \multirow{4}{*}{$\mathrm{D}$} & Proteína & $28.55 \pm 0.32$ & 31 & 7.90 & $<0.0001$ \\
& Grasa & $17.90 \pm 2.06$ & 30 & 40.33 & $<0.0001$ \\
& Lactosa & $46.94 \pm 0.47$ & 48 & 2.29 & 0.0001 \\
\hline \multirow{4}{*}{ E } & Proteína & $28.44 \pm 0.14$ & 31.2 & 8.84 & $<0.0001$ \\
& Grasa & $25.55 \pm 2.04$ & 30 & 14.83 & 0.0001 \\
& Lactosa & $47.21 \pm 0.17$ & 48 & 1.64 & $<0.0001$ \\
\hline \multirow{2}{*}{ F } & Proteína & $27.70 \pm 0.87$ & 32.1 & 15.26 & $<0.0001$ \\
& Grasa & $27.61 \pm 0.20$ & 32.1 & 13.98 & $<0.0001$ \\
& Lactosa & $46.44 \pm 0.56$ & 48 & 3.25 & $<0.0001$ \\
\hline \multirow{2}{*}{$\mathrm{G}$} & Proteína & $27.91 \pm 0.32$ & 32 & 12.78 & $<0.0001$ \\
& Grasa & $26.22 \pm 0.36$ & 30 & 12.60 & $<0.0001$ \\
& Lactosa & $47.38 \pm 0.28$ & 47 & -0.80 & 0.0737 \\
\hline \multirow{2}{*}{$\mathrm{H}$} & Proteína & $28.56 \pm 0.11$ & 31 & 7.87 & $<0.0001$ \\
& Grasa & $27.86 \pm 0.25$ & 30 & 7.13 & $<0.0001$ \\
& Lactosa & $47.64 \pm 0.08$ & 50 & 4.72 & $<0.0001$ \\
\hline
\end{tabular}

Los valores son la media \pm desviación estándar. Valor $-p<0.05$ indica diferencias estadísticas significativas entre valor experimental y el valor de referencia. 
último las marcas A y C (12.5\%). Los parámetros en los que incumplen son el contenido de proteína, caseína, grasa, sólidos no grasos, punto crioscópico y densidad. Resultados similares fueron reportados por el COFOCALEC (2006), en un estudio sobre el cumplimiento de la normatividad de los productos lácteos en el territorio nacional. Por lo que, los organismos encargados de verificar el cumplimiento de la normatividad oficial en productos comerciales deberían prestar mayor atención para que las empresas comercializadoras cumplan con los requisitos mínimos estipulados por las regulaciones (Tolentino-Mayo et al., 2018).

Cuadro 6. Cumplimiento de los parámetros de calidad en leche entera pasteurizada con la NOM-151-SCFI-2010.

\begin{tabular}{|c|c|c|c|c|c|c|c|c|c|}
\hline \multirow{2}{*}{ Marca } & \multicolumn{8}{|c|}{ Parámetros de calidad } & \multirow{2}{*}{$\begin{array}{c}\text { Cumplimiento } \\
(\%)\end{array}$} \\
\hline & Proteína & Caseína & Grasa & Lactosa & SNG & AT & $\mathrm{PC}$ & Densidad & \\
\hline A & No & No & No & No & No & $\mathrm{Si}$ & No & No & 12.5 \\
\hline B & No & No & No & $\mathrm{Si}$ & No & $\mathrm{Si}$ & No & No & 25 \\
\hline $\mathrm{C}$ & No & No & No & $\mathrm{Si}$ & No & $\mathrm{Si}$ & No & No & 12.5 \\
\hline $\mathrm{D}$ & No & No & No & $\mathrm{Si}$ & No & $\mathrm{Si}$ & No & $\mathrm{Si}$ & 37.5 \\
\hline E & No & No & No & $\mathrm{Si}$ & No & $\mathrm{Si}$ & No & $\mathrm{Si}$ & 37.5 \\
\hline $\mathrm{F}$ & No & No & No & $\mathrm{Si}$ & No & $\mathrm{Si}$ & No & $\mathrm{Si}$ & 37.5 \\
\hline $\mathrm{G}$ & No & No & No & $\mathrm{Si}$ & No & $\mathrm{Si}$ & No & $\mathrm{Si}$ & 37.5 \\
\hline $\mathrm{H}$ & No & No & No & $\mathrm{Si}$ & No & $\mathrm{Si}$ & No & $\mathrm{Si}$ & 37.5 \\
\hline
\end{tabular}

\section{Análisis clúster jerárquico}

Este tipo de análisis establece la relación (similitudes o diferencias) existente entre las marcas comerciales de leche entera pasteurizada (Falguera et al., 2011). En la Figura 1 se puede observar la generación de cuatro grupos (g1 a g4) de acuerdo con sus atributos nutrimentales y fisicoquímicos, el grupo g1 se conforma por la marca A, el grupo g2 por la marca $\mathrm{D}$, el grupo $\mathrm{g} 3$ por las marcas $\mathrm{C}$ y $\mathrm{B}$, mientras que el grupo g4 por las marcas H, G, F y E. Este resultado sugiere que existe variabilidad en las características nutrimentales y fisicoquímicas entre las marcas de leche evaluadas. Este tipo de análisis multivariado ha sido aplicado con éxito para la diferenciación o similitud entre marcas comerciales de leche entera pasteurizada (Juárez-Barrientos et al., 2015) y otros productos alimenticios (Falguera et al., 2011).

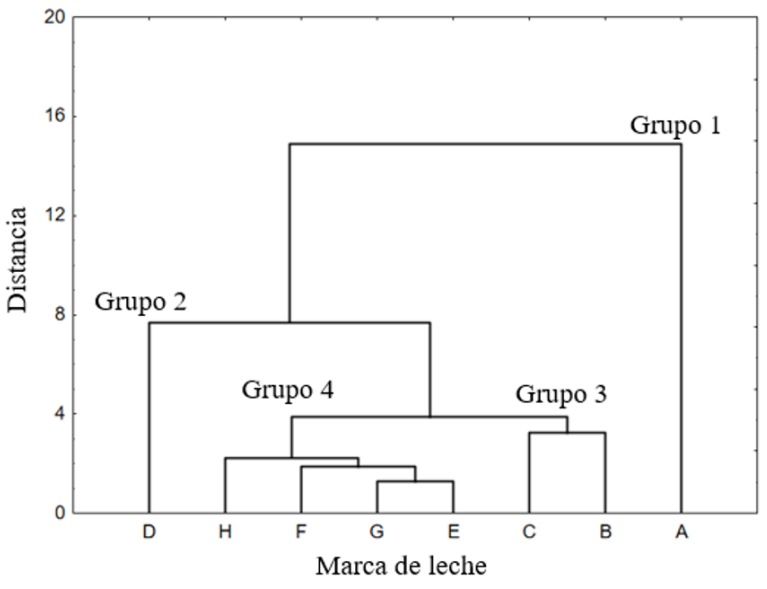

Figura 1. Dendograma de los grupos de leche entera pasteurizada.
Por su parte, en el Cuadro 7 se presentan las características nutrimentales y fisicoquímicas de los grupos obtenidos en el análisis clúster; además, se clasificó la calidad de la leche en inaceptable, mala, regular y aceptable de acuerdo con el cumplimiento normativo mínimo del contenido de proteína y grasa, además de considerar los valores del punto crioscópico y densidad de la leche (NOM-151-SCFI-2012). El grupo g1 (marca A), presentó un contenido de proteína y grasa de 21.95 y $23.83 \mathrm{~g} / \mathrm{L}$, es decir, concentraciones $27 \%$ y $21 \%$ menores a lo estipulado en la normatividad oficial, con valores de punto crioscópico de $-0.36^{\circ} \mathrm{C}$ y densidad de $1020 \mathrm{~g} / \mathrm{L}$, lo que la ubica como leche con calidad inaceptable. Por su parte, el grupo g2 (marca D) muestra una calidad mala con contenido proteico de $28.55 \mathrm{~g} / \mathrm{L}$ ( $<5 \%$ a la NOM) y graso de $17.9 \mathrm{~g} / \mathrm{L}$ (< $40 \%$ a la $\mathrm{NOM}$ ) con punto crioscópico de $-0.48^{\circ} \mathrm{C}$ y densidad de 1030 g/L. Asimismo, el grupo g3 (marcas C y B) muestra calidad regular tomando como referencia el contenido de proteína $(26.35 \mathrm{~g} / \mathrm{L})$ y grasa $(28.56 \mathrm{~g} / \mathrm{L})$ con valores bajos en el punto crioscópico $\left(-0.45^{\circ} \mathrm{C}\right)$ y densidad $(1026 \mathrm{~g} / \mathrm{L})$ en comparación con la normatividad oficial vigente $\left(-0.530^{\circ} \mathrm{C}\right.$ y $1029 \mathrm{~g} / \mathrm{L}$ ). Por último, el grupo g4 (marcas H, F, G y E) muestra una calidad aceptable (pero no buena) de acuerdo con su contenido de proteína $(28.15 \mathrm{~g} / \mathrm{L})$ y grasa $(26.81 \mathrm{~g} / \mathrm{L})$ que son ligeramente menores a lo establecido en la normatividad (30 g/L mínimo) con punto crioscópico de $-0.48^{\circ} \mathrm{C}$ y densidad de $1029 \mathrm{~g} / \mathrm{L}$, valores superiores $(\mathrm{p}<0.05)$ a los observados en los grupos g1, g2 y g3. Tal como se mencionó previamente, se requiere un mayor control de calidad en los procesos de las empresas y una mayor vigilancia por parte de las autoridades con la finalidad de evitar fraudes (intencionales o no) a los consumidores de este tipo de productos.

Cuadro 7. Características de los grupos obtenidos con base en el análisis clúster.

\begin{tabular}{ccccc}
\multirow{2}{*}{ Parámetros } & \multicolumn{4}{c}{ Grupos } \\
\cline { 2 - 5 } & $\mathrm{g} 1$ & $\mathrm{~g} 2$ & $\mathrm{~g} 3$ & $\mathrm{~g} 4$ \\
\hline Proténa $(\mathrm{g} / \mathrm{L})$ & $21.95 \pm 3.70^{\mathrm{c}}$ & $28.55 \pm 0.32^{\mathrm{a}}$ & $26.35 \pm 0.71^{\mathrm{b}}$ & $28.15 \pm 0.58^{\mathrm{a}}$ \\
Caseína $(\mathrm{g} / \mathrm{L})$ & $12.73 \pm 4.45^{\mathrm{c}}$ & $20.20 \pm 0.35^{\mathrm{a}}$ & $18.21 \pm 0.06^{\mathrm{b}}$ & $20.41 \pm 0.60^{\mathrm{a}}$ \\
Grasa $(\mathrm{g} / \mathrm{L})$ & $23.83 \pm 4.20^{\mathrm{d}}$ & $17.9 \pm 2.06^{\mathrm{c}}$ & $28.56 \pm 0.62^{\mathrm{a}}$ & $26.81 \pm 1.39^{\mathrm{b}}$ \\
Lactosa $(\mathrm{g} / \mathrm{L})$ & $37.16 \pm 4.89^{\mathrm{c}}$ & $46.94 \pm 0.47^{\mathrm{a}}$ & $43.85 \pm 0.91^{\mathrm{b}}$ & $47.12 \pm 0.55^{\mathrm{a}}$ \\
$\mathrm{SNG}(\mathrm{g} / \mathrm{L})$ & $58.88 \pm 10.34^{\mathrm{b}}$ & $78.65 \pm 0.99^{\mathrm{a}}$ & $72.27 \pm 1.75^{\mathrm{c}}$ & $77.95 \pm 1.23^{\mathrm{a}}$ \\
$\mathrm{AT}(\mathrm{g} / \mathrm{L})$ & $1.89 \pm 0.13^{\mathrm{a}}$ & $1.57 \pm 0.11^{\mathrm{c}}$ & $1.77 \pm 0.04^{\mathrm{b}}$ & $1.44 \pm 0.09^{\mathrm{d}}$ \\
PC $\left({ }^{\circ} \mathrm{C}\right)$ & $-0.365 \pm 0.064^{\mathrm{c}}$ & $-0.486 \pm 0.005^{\mathrm{a}}$ & $-0.451 \pm 0.011 \mathrm{~b}$ & $-0.488 \pm 0.009^{\mathrm{a}}$ \\
Densidad $(\mathrm{g} / \mathrm{L})$ & $1020.88 \pm 4.01^{\mathrm{c}}$ & $1030.55 \pm 0.72^{\mathrm{a}}$ & $1026.50 \pm 0.92^{\mathrm{b}}$ & $1029.61 \pm 0.64^{\mathrm{a}}$ \\
Marcas de leche & $\mathrm{A}$ & $\mathrm{D}$ & $\mathrm{C}, \mathrm{B}$ & $\mathrm{H}, \mathrm{F}, \mathrm{G}, \mathrm{E}$ \\
\hline Los valores son la media \pm desviación estándar. Letras diferentes por fila indican diferencias \\
estadísticas significativas $(p<0.05)$. SNG: sólidos no grasos; AT: acidez titulable; PC: punto \\
crioscópico.
\end{tabular}

\section{Conclusiones}

De acuerdo con la evidencia, existen diferencias en los parámetros fisicoquímicos entre los distintos lotes de producción de la misma marca comercial, además de las diferencias entre marcas comerciales. Asimismo, ninguna de las marcas cumple con lo reportado en su etiqueta (proteína, grasa y lactosa) ni con los criterios de calidad estipulados en la NOM-151-SCFI-2012 para leche entera pasteurizada. Lo anterior, demuestra la necesidad e importancia de monitorear la calidad de los productos comerciales, con la finalidad de evitar que se cometan fraudes. Además, se requiere mayor vigilancia por parte de las autoridades correspondientes hacía las empresas del ramo, para asegurar el cumplimiento de los 
parámetros establecidos por la normatividad oficial vigente y permitan al consumidor obtener productos lácteos de buena calidad.

\section{Agradecimientos}

Los autores agradecen a las autoridades del Centro Universitario de los Altos de la Universidad de Guadalajara. Particularmente, al Dr. Sergio Franco Casillas (Jefe del Departamento de Ingenierías) por su valioso apoyo para realizar este proyecto.

\section{Literatura citada}

- AOAC. (1990). Official methods of análisis of Association Official of Agricultural Chemists International. AOAC international, Gaithersburg, Maryland, USA.

- AOAC. (2005). Official methods of análisis of Association Official of Agricultural Chemists International. AOAC international, Washington, DC, USA.

- Arriaga, A., A. Guzmán, A. Morales, B. Olivares, E. Ramírez-Moreno y J.A. Ariza-Ortega. (2019). Evaluación de la información nutrimental del etiquetado del yogurt natural y griego. Educación y Salud Boletín Científico de Ciencias de la Salud del ICSa, 7(14), 28-31. https://doi.org/10.29057/icsa.v7i14.4452

- Awan, A., M. Naseer, A. Iqbal, M. Ali, R. Iqbal, y F. Iqbal. (2014). A study on chemical composition and detection of chemical adulteration in tetra pack milk samples commercially available in Multan, Pakistan. Pakistan Journal of Pharmaceutical Sciences, 27(1), 183186.

- Bermúdez-Aguirre, D., R. Mawson, K. Versteeg y G.V. Barbosa-Cánovas. (2009). Composition properties, physicochemical characteristics and shelf life of whole milk after thermal and thermo sonication treatments. Journal of Food Quality, 32(3), 283-302. https://doi.org/10.1111/j.1745-4557.2009.00250.x

- Briñez, W.F., E. Valbuena, G. Castro, F. Fuentes, D. González y A. Tovar. (2002). Physical and chemical quality of main milk brands user sold in the city of Maracibo. Revista Científica, FCV-LUZ, 7(3), 221-230.

- Calderón, A., V. Rodríguez y S. Vélez. (2007). Evaluación de la calidad composicional en cuatro procesadoras de quesos en el municipio de Montería, Colombia. Revista MVZ Córdoba, 12(1), 912-921.

- CEDRSSA (Centro de Estudios para el Desarrollo Rural Sustentable y la Soberanía Alimentaria). (2019). Reporte Situación de la ganadería lechera en el sureste de México. [publicación en línea]. Disponible desde Internet en <http://www.cedrssa.gob.mx/files/b/13/19Ganader\%C3\% ADa-Lechera.pdf>. [consultado 25 marzo 2021, 19:33].

- COFOCALEC (Consejo para el Fomento y Calidad de Leche). (2006). Estudio sobre el cumplimiento de la NOM-151-SCFI-2004 de los productos de leche en el territorio nacional. [publicación en línea]. Disponible desde Internet en <

http://www.cofocalec.org.mx/cofocalec.php?

script=sci_arttext\&pid=S2007-11242013000100006>.

[consultado 19 enero 2021, 13:18].

- Dirección de Investigación y Evaluación Económica y Sectorial (2019). Panorama Agroalimentario Leche y Lácteos. FIRA Fideicomisos Instituidos en Relación con la Agricultura [publicación en línea]. Disponible desde Internet en <https://www.inforural.com.mx/wpcontent/uploads/2019/06/Panorama-Agroalimentario-

Leche-y-la769cteos-2019.pdf>. [consultado 25 marzo 2021, 19:21].

- Elika. (2011). Fraudes alimentarios. Berezi@ 26. [publicación en línea]. Disponible desde <file:///C:/Users/Propietario/Downloads/Fraudes\%20alim entarios\%20ELIKA.pdf>. [consultado 25 marzo 2021, 18:32].

- Falguera, V., A.M. Sánchez-Riaño, J.P. Quintero-Cerón, C.A. Rivera-Barrero, J.J. Méndez-Arteaga y A. Ibartz. (2011). Characterization of polyphenol oxidase activity in juices from 12 underutilized tropical fruits with high agroindustrial potential. Food and Bioprocess Technology, 5, 2921-2927. DOI 10.1007/s11947-0110521-y

- Feria-Cuevas, Y., P. Landeros-Ramírez, Z. Gómez-Cruz y J.L. Soriano-Ureña. (2019). Hábitos de consumo de productos lácteos, pasteurizados y no pasteurizados, en una población universitaria. e-CUCBA, 12(6), 49-59. https://doi.org/10.32870/e-cucba.v0i12.137

- Galván, M.P. (2005). Proceso básico de la leche y el queso. Revista Digital Universitaria, 6(9), 2-17.

- Gandy, A.L., M.W. Schilling, P.C. Coggings, C.H. White, Y. Yoon y V.V. Kamadia. (2008). The effect of pasteurization temperature on consumer acceptability, sensory characteristics, volatile compound composition, and shelf-life of fluid milk. Journal of Dairy Science, 91(5), 1769-1777. https://doi.org/10.3168/jds.2007-0833

- Juárez-Barrientos, J.M., J. Rodríguez-Miranda, C.E. Martínez-Sánchez, B. Hernández-Santos, E. Paz-Gamboa, C.A. Gómez-Aldapa, P. Díaz-Rivera y E. Hernan-Lara. (2015). Evaluación y caracterización de calidad de leches comerciales consumidas en Tuxtepec, Oaxaca, México. Ecosistemas y Recursos Agropecuarios, 2(6), 327-337.

- Negri, L.M. (2005). El pH y la acidez de la leche. pp. 155-161. En: Tavera, M., L. Calvinho, R. Páez, M. Chavez, V. Charlón, V. Vanzini, A. Abdala, A. Castillo, A. Cuatrin (Eds.), Manual de referencias técnicas para el logro de leche de calidad. INTA Rafaela, Buenos Aires, Argentina.

- Nicolau, N., Y, Xu y R. Goodacre. (2011). MALDI-MS and multivariate analysis for the detection and quantification of different milk species. Analytical and Bioanalytical Chemistry, 399(10), 3491-3502. 10.1007/s00216-011-4728-6

- Noa-Pérez, M., M. Cortés-Marín, P. Landeros-Ramírez, Z. Gómez-Cruz, M. Real-Navarro, R. Reynoso-Orozco, T.J. 
Jaime-Ornelas y C. Juárez-Woo. (2021). Límites de detección de algunos métodos de prueba para adulterantes e inhibidores en leche. e-CUCBA, 8(15), 33-43. https://doi.org/10.32870/e-cucba.v0i15.177

- Noa-Pérez, M., P. Landeros-Ramírez, Z. Gómez-Cruz, D.G. González-Aguilar, M. Real-Navarro, M.S. MedinaLerena y R. Reynoso-Orozco. (2019). Incidencia de adulterantes en leches cruda y pasterizada en el estado de Jalisco, México. e-CUCBA, 6(12), 15-28. https://doi.org/10.32870/e-cucba.v0i12.133

- Norma Oficial Mexicana. NOM-051-SCFI-2010. Información comercial, disposiciones generales para productos. Secretaria de Comercio y Fomento Industrial. Dirección General de Normas. Estados Unidos Mexicanos. DOF [publicación en línea]. Disponible desde Internet en <http://www.dof.gob.mx/nota_detalle.php? codigo $=4867159 \&$ fecha $=24 / 01 / 1996>$. [consultado 25 marzo 2021, 18:57].

- Norma Oficial Mexicana. NOM-091-SSA1-1994. Bienes y Servicios. Leche pasteurizada de vaca. Disposiciones y especificaciones sanitarias. DOF [publicación en línea]. Disponible desde Internet en < http://dof.gob.mx/nota_detalle.php? codigo $=4756611 \&$ fecha $=27 / 10 / 1994 \&$ print $=$ true $>$. [consultado 25 marzo 2021, 18:58].

- Norma Oficial Mexicana. NOM-155-SCFI-2012. LecheDenominaciones, especificaciones fisicoquímicas, información comercial y métodos de prueba. DOF [publicación en línea]. Disponible desde Internet en <http://www.dof.gob.mx/normasOficiales/4692/seeco/see co.htm>. [consultado 25 marzo 2021, 19:00].

- PROFECO (Procuraduría Federal del Consumidor). (2004). No todo lo que parece leche lo es. Leche, fórmulas lácteas y productos lácteos combinados. Consumidor [publicación en línea]. Disponible desde Internet en $<$ https://docplayer.es/12095753-No-todo-lo-que-pareceleche-lo-es.html>. [consultado 25 marzo 2021, 19:04].

- Reyes, J., F. Bon, J. Moreno, C. Rubio, A. Valdivia. (2007). Adulteración de leche pasteurizada con suero de quesería en la ciudad de Aguascalientes. Avances en Investigación Agropecuaria, 11(2), 23-34.

- Rondón, L., M. Lara e I. González. (2003). Agentes adulterantes y conservadores en la leche fluida. Revista de la Facultad de Farmacia, 45(2), 45-50.

- Secretaría de Economía. (2020). Economía ordena suspensión inmediata de la comercialización de productos denominados como "queso" y "yogurt natural" que incumplen con NOMs. Secretaría de Economía - Blog [publicación en línea]. Disponible desde Internet en $<$ https://www.gob.mx/se/articulos/economia-ordenasuspension-inmediata-de-la-comercializacion-deproductos-denominados-como-queso-y-yogurt-naturalque-incumplen-con-noms?idiom=es $>$. [consultado 25 marzo 2021, 19:29].

- CANILEC (Cámara Nacional de la Industria de la Leche). (2021). Estadísticas del sector lácteo. [publicación en lí- nea]. Disponible desde Internet en < https://www.canilec.org.mx/estadisticas-2/>. [consultado 17 de junio 2021, 20:50].

- Tolentino-Mayo, L., S. Rincón-Gallardo, L. BahenaEspina, V. Ríos y S. Barquera. (2018). Conocimiento y uso del etiquetado nutrimental de alimentos y bebidas industrializadas en México. Salud Pública de México, 60(3), 328-337. https://doi.org/10.21149/8825 